\title{
Gut-associated lymphoid tissue contains the molecular machinery to support T-cell-dependent and T-cell-independent class switch recombination
}

\author{
F Barone ${ }^{1}$, P Patel $^{1,2}$, JD Sanderson $^{2}$ and J Spencer ${ }^{1}$
}

\begin{abstract}
A PRoliferation-Inducing Ligand (APRIL) is a secreted cytokine member of the tumor necrosis factor family. It is a B-cell survival factor that also induces class switch recombination (CSR) toward immunoglobulin A (IgA), independent of $\mathrm{T}$ cells. It is therefore an important contributor to the maintenance of the mucosal immunological barrier, which has been linked to a putative extrafollicular inductive phase of the IgA response in lamina propria. By immunohistochemistry (IHC) and quantitative real-time PCR (qRT-PCR) on microdissected tissue from normal human gut, we observed APRIL expression, together with TACI (transmembrane activator and CAML interactor) and BCMA (B-cell maturation antigen), in gut-associated lymphoid tissue (GALT), lamina propria, and in the epithelium of stomach, small and large intestine, and rectum. However, no activation-induced cytidine deaminase (AID) expression (an absolute requirement for class switching) was detected in lamina propria by IHC or qRT-PCR. APRIL and its receptors were only observed alongside AID in GALT, showing that GALT contains the apparatus to support both T-independent and T-dependent routes to IgA CSR.
\end{abstract}

\section{INTRODUCTION}

A PRoliferation-Inducing Ligand (APRIL) is a pluripotent cytokine belonging to the tumor necrosis factor family that was initially described as a trophic factor for epithelial cells and has been associated with malignant epithelial proliferation. ${ }^{1}$ More recently, APRIL has been shown to exert an additional effect in the homeostasis of antibody-producing cells. Animals defective for APRIL or its receptors, TACI (transmembrane activator and CAML interactor) and BCMA (B-cell maturation antigen), have impaired plasma cell survival and defects in immunoglobulin A (IgA) and IgM production ${ }^{2,3}$ It has also been shown that APRIL is a potent IgA switch factor that can induce the expression of activation-induced cytidine deaminase (AID), an essential prerequisite for class switch recombination (CSR). APRILinduced CSR is independent of the interaction between CD40 and CD40L or TGF $\beta,{ }^{4}$ and this route to IgA CSR is therefore $\mathrm{T}$-cell independent and potentially extrafollicular. These data have been used to support the concept that lamina propria is involved in an inductive phase of mucosal IgA response.
However, this has proven to be contentious, partly because, to date, there is no consensus on AID expression in lamina propria. ${ }^{5-7}$ The study that initially proposed that AID was expressed in murine lamina propria ${ }^{8}$ failed to take the contribution of isolated lymphoid follicles to cell isolates into account. ${ }^{9,10}$ The critical contribution of isolated lymphoid follicles to IgA production in mice has been highlighted recently. ${ }^{11}$ Moreover, the B1 B cells that contribute to the lamina propria plasma cell population in mice ${ }^{12}$ have not been detected in humans. ${ }^{13}$ It is important to understand the relative importance of gut-associated lymphoid tissue (GALT) and lamina propria as immunological inductive sites for IgA production in humans. Efforts to target mucosal vaccines require this key information.

A PRoliferation-Inducing Ligand and its receptors were shown to regulate the last phases of $\mathrm{B}$ lineage maturation in vitro. The upregulation of TACI (TNFRSF13) and BCMA (TNFRSF17) on antibody-secreting cells ${ }^{14}$ and the presence of APRIL within the plasma cell survival niche in the bone marrow ${ }^{15}$ support the role of this network in plasma cell homeostasis. An equivalent role

\footnotetext{
${ }^{1}$ Peter Gorer Department of Immunobiology, King's College London, London, UK. ²Nutritional Sciences Division, King's College London, London, UK. Correspondence: J Spencer (jo.spencer@kcl.ac.uk)

Received 2 July 2009; accepted 2 July 2009; published online 9 September 2009. doi:10.1038/mi.2009.106
} 
for APRIL derived from neutrophils in inflammatory conditions in the gut is suggested. ${ }^{16}$

We therefore undertook a study of the expression of APRIL, its receptors TACI and BCMA, as well as AID, in the context of the microanatomical organization of the human gastrointestinal tract. We also studied APRIL expression in the stomach in Helicobacter Pylori gastritis, in which the epithelium that is rich in Toll-like receptor 5 (TLR5) becomes exposed to flagellated bacteria, the context in which APRIL might be induced on colonic epithelium.

By immunohistochemistry (IHC) and quantitative real-time PCR (qRT-PCR) on microdissected tissue from normal human gut, we observed APRIL expression, together with TACI and BCMA, in GALT, lamina propria, and in the epithelium of small and large intestine. Our data do not support the hypothesis that epithelial APRIL is induced by bacterial ligands or that it promotes CSR in lamina propria; no AID expression is detected in lamina propria by IHC or qRT-PCR. However, we found that APRIL and its receptors exist alongside AID in GALT, showing that GALT contains the apparatus to support both T-independent and T-dependent routes to IgA CSR.

\section{RESULTS}

\section{Protein and mRNA expression of APRIL in GALT}

All gut samples evaluated showed APRIL expression in the GALT, predominantly as punctate staining in the cytoplasm of large cells. An intense APRIL expression was observed within the T-cell zone and in the dome area facing the gut lumen (Figure 1A and B, brown). Double staining with CD68 and CD11c showed that the majority of APRIL + cells in this area were macrophages and dendritic cells (DCs) (Figure 1B). APRIL expression was detected in the germinal center (GC), within CD68 + tingible body macrophages (Figure 1C, CD68 in pink and APRIL in brown, also as single in the inset).

Within the T-cell zones (Figure 1D), the vessels, high endothelial venules (Figure 1D asterisk), and DCs expressed APRIL. Double staining with APRIL and CD20 showed that a close cellular contact between APRIL + cells and CD20 + cells was often present but no CD20 + /APRIL + cells were identified (data not shown). The epithelium above the lymphoid aggregates was consistently positive for APRIL (Figure 1B). APRIL was consistently detected in the T-cell zone and in GC tingible body macrophages and on the stratified epithelium in control tonsil tissue (data not shown).

We performed qRT-PCR on whole microdissected lymphoid tissue from frozen ileum and colon (Figure 1E and F), and we compared the levels of expression of APRIL and its receptors, TACI and BCMA, with the expression of the same molecules in the GC of tonsils. APRIL, TACI, and BCMA expression levels in gut lymphoid tissue did not significantly differ from the expression of the same molecules in the tonsil (Figures 1a-c).

\section{Relative distribution of APRIL and AID in protein and mRNA levels in GALT}

In all the samples examined, AID protein expression was consistently detected within the dark zone of GALT GCs (Figure 2A).
Large interfollicular B cells with AID in their cytoplasm were also detected at the periphery of lymphoid tissue (Figure 2B). This distribution of AID closely resembles the expression of this molecule in secondary and tertiary lymphoid organs. ${ }^{17}$

Appendix, ileum, colon, and tonsil samples were double stained for APRIL and AID. Close proximity of cells expressing the two molecules was observed within the organized lymphoid tissue, predominantly in the GC (Figure $\mathbf{2 C}$ and $\mathbf{D}$, respectively, tonsil and ileum). In contrast to other studies, the lymphocyte population adjacent to and within the epithelium, including the stratified lymphoepithelium of tonsils that was consistently strongly positive for APRIL, did not include AID-expressing cells (Figure 2E and $\mathbf{F}$ ).

Using qRT-PCR on microdissected lymphoid tissue, we showed that the relative amount of AID transcripts in the GALT GC of both ileum and colon was significantly less than the relative number in tonsil GC (Figure 2a).

\section{APRIL expression in the lamina propria}

An intense APRIL expression was detected in the lamina propria of the samples evaluated. APRIL was detected in the cytoplasm of a large number of cells localized beneath the gut epithelium (Figure 3A). The number of APRIL + cells and the intensity of APRIL staining were higher in the lamina propria closer to the lymphoid tissue (Figure $\mathbf{3 A}$ and insets). These areas were characterized by the presence of CD20 + cells at the periphery of the follicle (Figure 4B and insets). This phenomenon was consistently observed in areas close to ileal Peyer's patches and colonic follicles (Figure 3C).

Most APRIL-positive cells beneath the surface epithelium were CD68 + monocytes/macrophages (Figure 4D). The APRIL + / CD68 - cells were characterized by the expression of CD11c and DC morphology (Figure 3E). Relatively few APRIL + cells expressed neutrophil elastase (data not shown).

The relative number of APRIL mRNA transcripts in the microdissected lamina propria was highly variable, presumably because of the variable cellular composition of the microdissected samples. However, it was apparent that the relative number of APRIL mRNA transcripts in the microdissected lamina propria of the ileum and colon was significantly higher than the relative number detected in the tonsil (respectively, $P=0.0038$ and $P=0.0006$ ) (Figure 4a). We consider this to be associated with the relatively lower proportion of APRILexpressing cells in the densely cellular environment of lymphoid tissues, compared with that in the lamina propria. Both TACI and BCMA were detectable in the lamina propria of the ileum and colon with levels comparable with those observed in tonsil GC (Figure 3b).

\section{AID expression in the lamina propria of normal gut}

Sequential sections were stained for APRIL and for an anti-AID antibody, and a selection of samples of ileum and colon were double stained with APRIL/AID, AID/CD20, and AID/CD68. When double stained with CD20, we observed that none of the AID + cells in the lamina propria were CD20 + (Figure 4B and high magnification in the inset). Light microscopy reveals that 

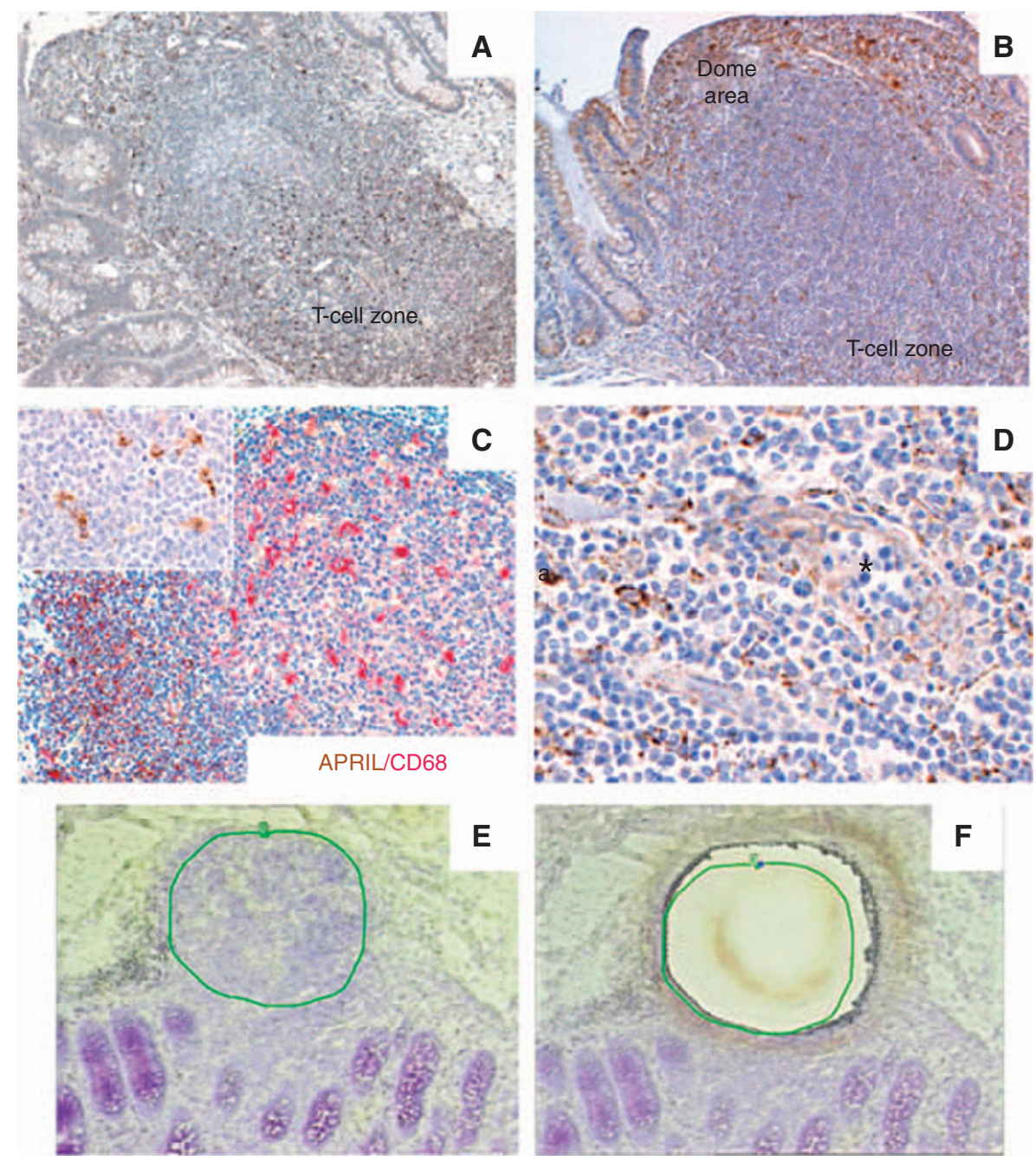

a

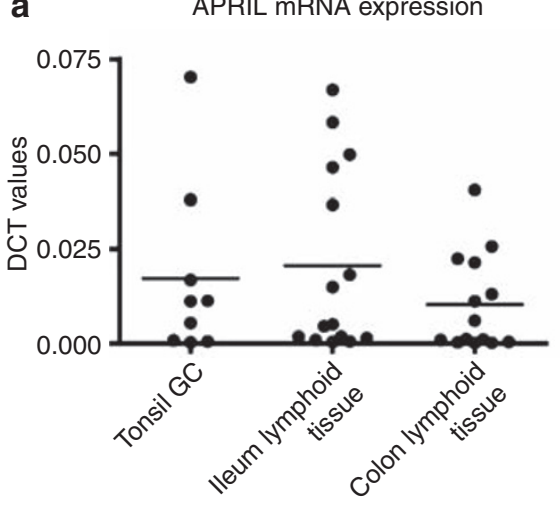

b

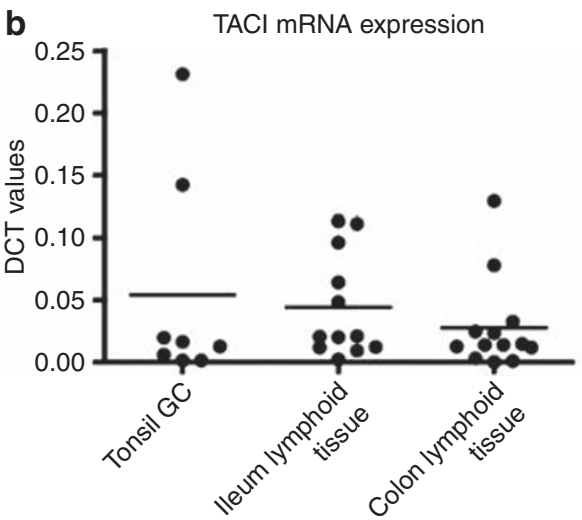

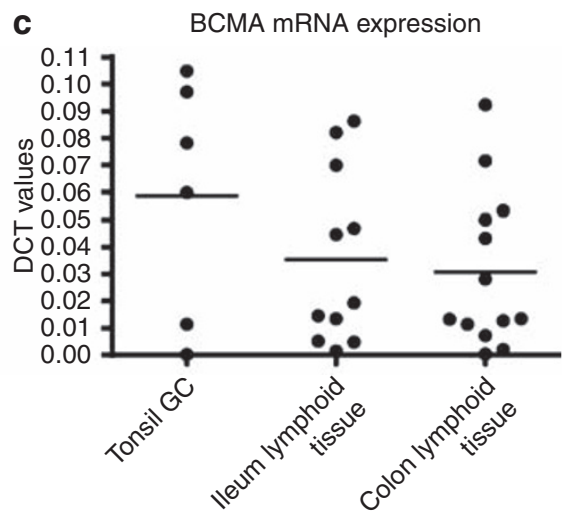

Figure 1 Distribution of A PRoliferation-Inducing Ligand (APRIL), transmembrane activator and CAML interactor (TACI), and B-cell maturation antigen (BCMA) in human gut-associated lymphoid tissue (GALT). Photomicrographs of $3 \mu \mathrm{m}$-thick sections selected from specimens of normal gut stained for APRIL (A-D) and double stained with CD68 (purple) (C) to evaluate APRIL distribution in human GALT. Single staining showing diffuse and reticular APRIL distribution in two lymphoid follicles in the digestive tract (A and B), with an intense APRIL staining in the T-cell zone and in the dome area, and a spotty discrete expression in the germinal center (GC) (A and B). Double staining showing colocalization between APRIL and CD68+ tingible body macrophages (pink) in GALT GCs (C, double staining; inset, single staining for APRIL). Within the T-cell zone, high endothelial venules (asterisk) and dendritic cells express APRIL (D). Photomicrographs of a GC from one of the samples of colon before (E) and after (F) laser capture microdissection. Diagram showing quantitative Taqman real-time PCR evaluation of APRIL (a), TACI (b), and BCMA (c) transcript levels $\left(\triangle C_{t}[D C T]\right)$ on microdissected tissue from ileum and colon samples, and dissected GCs from human tonsils used as standard control. Transcript levels of APRIL, TACI, and BCMA in ileum and colon lymphoid tissue were not statistically different from those observed in the tonsil. 

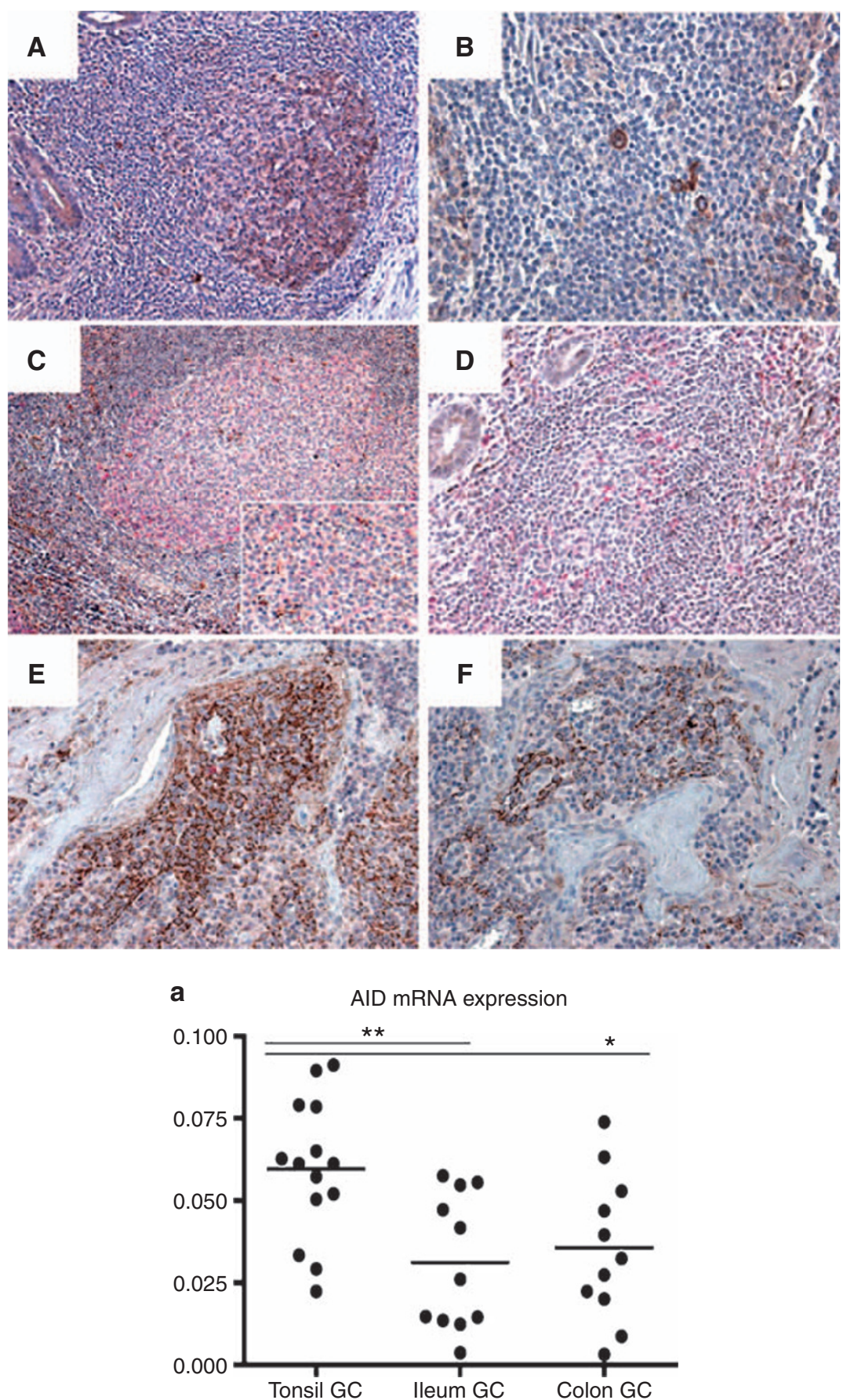

Figure 2 Relationship between activation-induced cytidine deaminase (AID) and A PRoliferation-Inducing Ligand (APRIL) expression in the lymphoid tissue of the gut and tonsil. Examples of immunohistochemistry for AID (brown in $\mathbf{A}$ and $\mathbf{B}$ and pink in $\mathbf{C}-\mathbf{F}$ ) in single (A and $\mathbf{B})$ and double staining with APRIL (brown C-F) performed on $3 \mu \mathrm{m}$-thick sections of two specimens of human gut and tonsil. Within the germinal center (GC) of the gut-associated lymphoid tissue (GALT), an intense AID expression was detected in the dark zone (A in brown and C pink) and in large interfollicular B cells (B). APRIL/AID double staining showed close contact of the two molecules within the GALT (C and D). In the tonsil, no AID+ cells were detected in areas close to the APRIL+ epithelium (brown in E and F). Quantitative real-time PCR was used to quantify AID expression in microdissected GC of ileum and colon in comparison with tonsil GC. (a) Lower level of AID was detected in GALT GC as compared with that in tonsils (ileum vs. tonsil ${ }^{* *} P<0.0025$ and colon vs. tonsil $\left.G C{ }^{*} P<0.0113\right)$.

the antibody most commonly used to detect AID in human tissue sections consistently binds to ceroid in CD68 + cells (Figure 4A, C and $\mathrm{D}$ and inset). Control rat antibody does not have the same property, as shown in the sequential section of tonsil stained with AID (Figure 4E and F).
We detected either no or negligible AID gene transcripts in lamina propria. We compared the relative numbers of APRIL and AID gene transcripts in the lymphoid tissue and in the lamina propria of both ileum and colon and we observed that, although APRIL mRNA transcripts were more abundant in the 

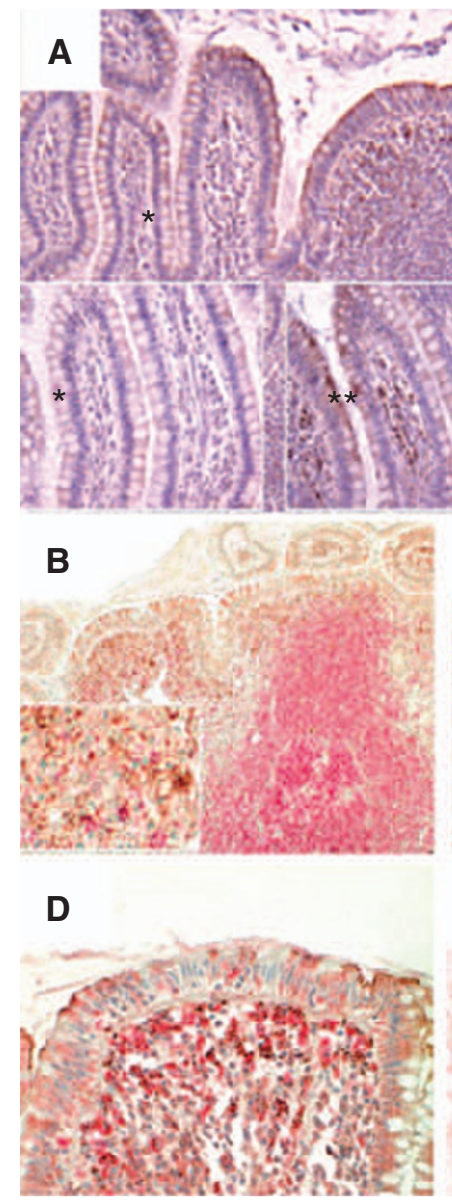
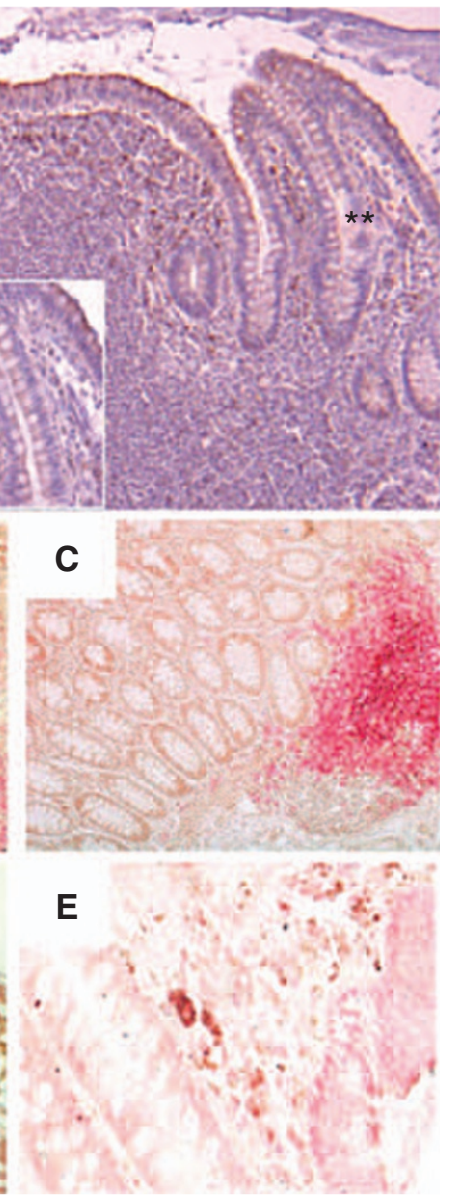
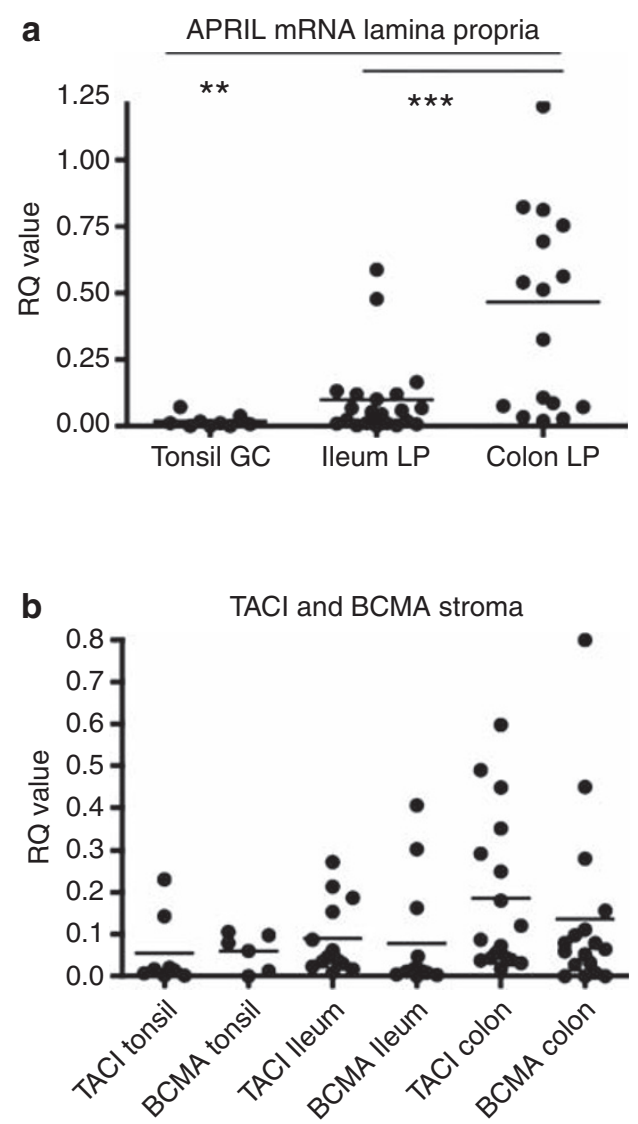

Figure 3 A PRoliferation-Inducing Ligand (APRIL), transmembrane activator and CAML interactor (TACI), and B-cell maturation antigen (BCMA) expression in the lamina propria of normal human gut. Immunohistochemistry for APRIL (brown) in single (A and inset), and in double staining (B-E) with CD20 (pink B and C), CD68 (pink in D), and CD11c (pink in E) on $3 \mu$ m-thick sections of three specimens of ileum (A, B, D) and two specimens of colon (C and E). (A) APRIL was diffusely expressed in the lamina propria of normal gut. A strong expression was detected in the areas beneath the epithelium, in particular in areas close to lymphoid tissue (double asterisks in the inset). (B and C) Double staining with CD20 showed a more intense APRIL expression in the proximity of lymphoid tissue. A higher magnification of the dome area showing closeness between CD20 + cells (pink) and APRIL + cells, although no APRIL/CD20-positive cells were seen. (D) The large majority of APRIL-positive cells in the lamina propria were macrophages (CD68 + in pink) and CD11c + cells (E). (a and b) Diagram showing quantitative Taqman real-time PCR evaluation of APRIL, TACI, and BCMA transcript levels in microdissected lamina propria. Significantly increased APRIL expression in the lamina propria of colon ( ${ }^{* \star} P=0.0038$ ) compared with that of control tonsil germinal center $(\mathrm{GC})$ and ileum lamina propria $\left({ }^{\star \star \star} P=0.0006\right)$. A similar trend with an increased expression of TACl and BCMA in colon lamina propria compared with that in the ileum $(P=$ n.s.) was observed.

colon and ileum lamina propria than in lymphoid tissue of the same organs, APRIL and AID were coexpressed only in lymphoid tissue and not in the lamina propria (Figure 4a).

Previous studies have detected AID expression in lamina propria. In those studies, AID + cells were adjacent to IgD + cells, indicative of the local presence of organized lymphoid tissue. ${ }^{7}$ We therefore identified areas of lamina propria containing sparse $\mathrm{CD} 20$ + cells that we considered to represent the periphery of lymphoid tissue, and we stained sequential sections of ileum and colon with cresyl violet for microdissection. We performed AID qRT-PCR with four different commercially available sets of primers and probes for AID on isolated areas, including sparse CD20 + cells and areas devoid of CD20 + cells. All four sets showed either no or a negligible expression of the molecule in the lamina propria, irrespective of the presence or absence of CD20 + cells (Figure 4b).

\section{APRIL protein and mRNA distribution in the epithelium of normal gut}

It has been shown that epithelial APRIL expression is induced by intestinal flora through TLR5 stimulation. ${ }^{7}$ We therefore assessed APRIL expression in gut epithelium. In samples of stomach (Figure 6a), duodenum, jejuneum, ileum (Figure 6b), colon (Figure $\mathbf{6 c}$ and $\mathbf{d}$ ), and rectum, we detected scarce or no expression of APRIL on the areas of epithelium adjacent to gut lumen, whereas an intense APRIL expression was detected in crypts (Figure 6b and c). An intense APRIL epithelial expression was detected in epithelial areas close to the lymphoid component (Figure 5d).

A PRoliferation-Inducing Ligand was first described as an epithelial proliferating factor, ${ }^{1}$ and a higher APRIL expression in crypts is in line with this function. This was further supported in the sample of gastric carcinoma evaluated that showed 

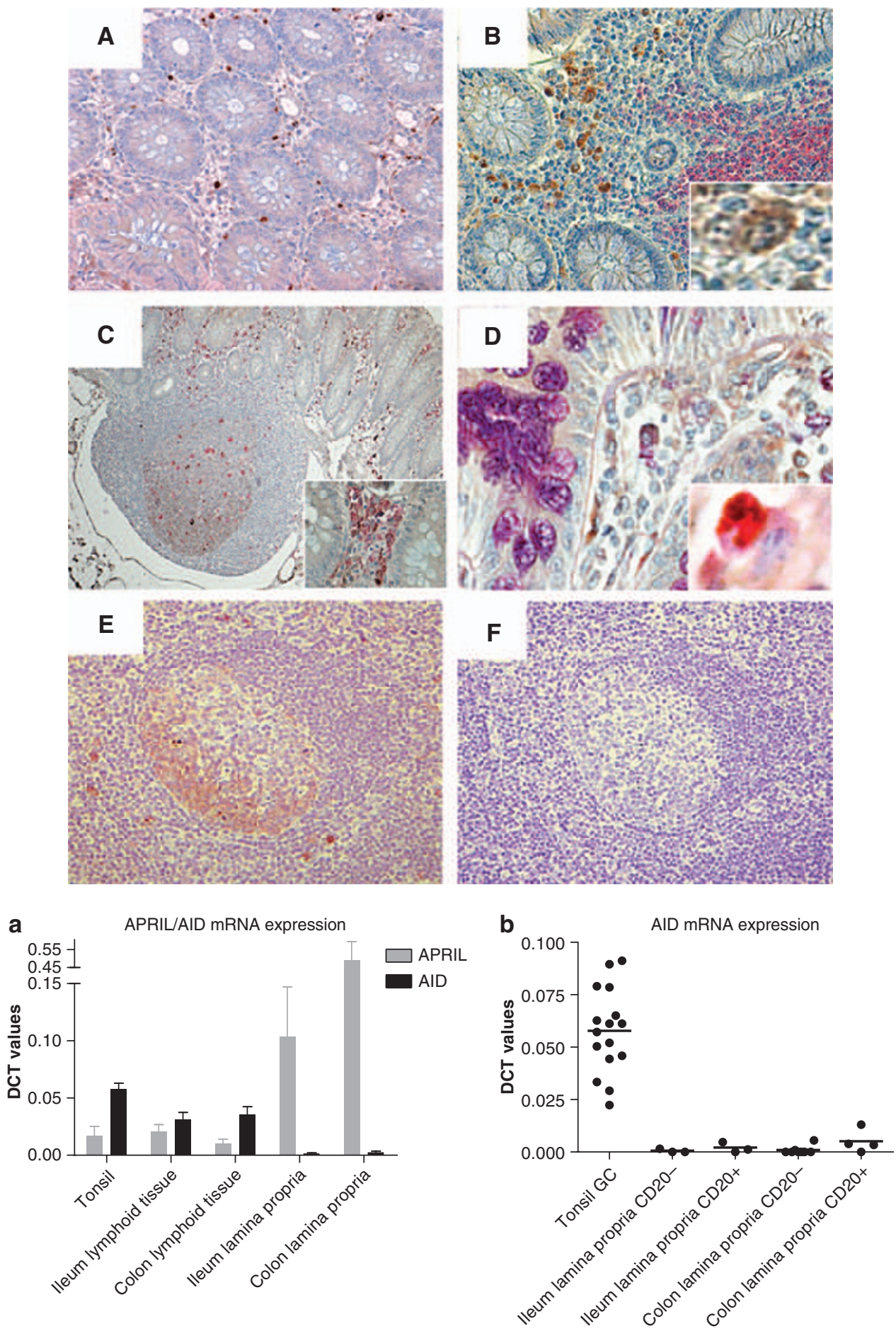

Figure 4 Activation-induced cytidine deaminase (AID) expression in gut lamina propria. Examples of AID expression in the lamina propria of normal gut and tonsil. Immunohistochemistry showing sparse cells in the lamina propria binding the anti-AID antibody in two diverse specimens of colon ( $\mathbf{A}$ and $\mathbf{B}$ ). These cells were shown to be negative for CD20 (B and higher magnification in the inset) and positive for CD68 (C and higher magnification in the inset). Periodic acid-Schiff staining showed that macrophages binding AID antibody were ceroid-loaded macrophages (D), as shown by sequential double staining with CD68 (inset). (E and F) Sequential staining of tonsil showing a typical AID distribution within the germinal centers (GCs) and in large interfollicular B cells (arrows), negative control with rat immunoglobulins. (a and b) Diagrams showing quantitative real-time PCR results for AID expression (gray bars) in microdissected tissue in comparison with A PRoliferation-Inducing Ligand (APRIL) expression levels (black bars). Biological replicates evaluated for APRIL and AID, respectively: Tonsil 9 and 16 (6 samples microdissected), ileal gut-associated lymphoid tissue (GALT) 15 and 11 (7 samples), colonic GALT 14 and 11 (10 samples), ileum lamina propria 16 and 7 ( 7 samples), colon lamina propria 16 and 12 (10 samples). Bars represent means \pm standard error (s.e.). In the lamina propria of both ileum and colon, despite a high expression of APRIL, no convincing AID expression was detected at the mRNA level. (b) Analysis on the mRNA expression of AID in areas of lamina propria with CD20 + cells and without CD20 + cells, showing that the presence of small aggregates of B cells did not influence the expression of AID, which was none or negligible as compared with that in lymphoid tissue (for the ileum and colon vs. tonsil GC $P<0.0002$ and $P<0.0001$, respectively). 


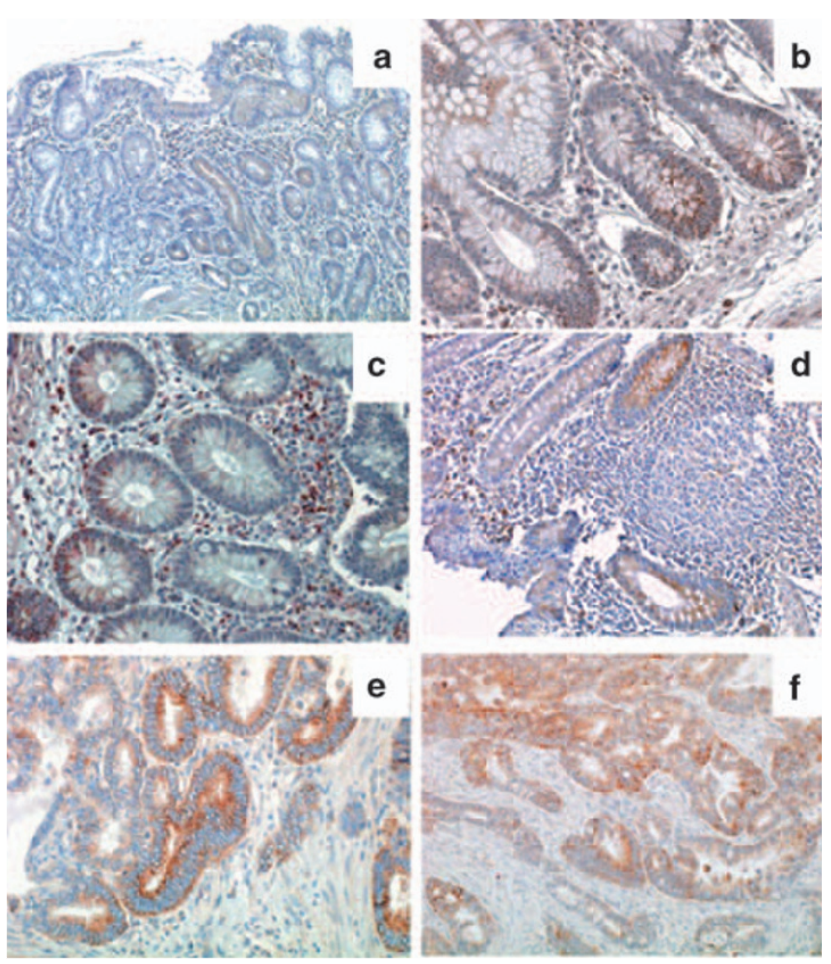

Figure 5 A PRoliferation-Inducing Ligand (APRIL) expression in the epithelium of normal gut. Immunohistochemistry for APRIL (brown) (a-f), performed on $3 \mu \mathrm{m}$-thick sections of stomach (a, e, and $\mathbf{f}$ ), ileum ( $\mathbf{b}$ and $\mathbf{d}$ ), and colon (c). Shown are the results for two stomach, two ileum, and one colon samples. (a) APRIL expression is scarce in normal gastric epithelium. (b and $\mathbf{c}$ ) On the base of crypts, both in the ileum and colon, an intense expression of the molecule was detected. (b-d) Of interest, no convincing luminal expression of APRIL was detected on the lumen-facing epithelium, with the exception of the epithelial areas close to organized lymphoid tissue (d). (e and f) Example of gastric carcinoma showing diffuse and intense APRIL expression, above the crypts, in proliferating epithelium.

a homogeneous and intense distribution of APRIL in proliferating malignant epithelium (Figure 5E and F).

\section{APRIL expression does not colocalize with H. Pylori in $\mathbf{H}$. Pylori-induced gastritis}

Gastric epithelium expresses TLR5, the innate receptor for flagellin, which is expressed by H. Pylori ${ }^{18}$ and is the previously proposed route for APRIL induction. ${ }^{7}$ We single and double stained sequential sections of stomach affected by $H$. pylori gastritis with APRIL and CD20; Giemsa staining was performed on sequential sections to detect the distribution of bacteria.

APRIL was detected in the epithelium of glandular structures (Figure 6a, asterisk), which were negative for $H$. pylori in a sequential section stained with Giemsa (Figure 6b). However, H. pylori were visualized in close contact with gastric epithelium around the neck of glands, in areas predominantly negative for APRIL (Figure $\mathbf{5 c}$ and $\mathbf{d}$ ). As observed in normal epithelium, the areas facing the lymphoid infiltrate showed an intense APRIL protein expression (Figure 5e and $\mathbf{f}$ ).

\section{DISCUSSION}

A PRoliferation-Inducing Ligand is known to regulate in vitro T-cell-independent CSR toward IgA, inducing AID

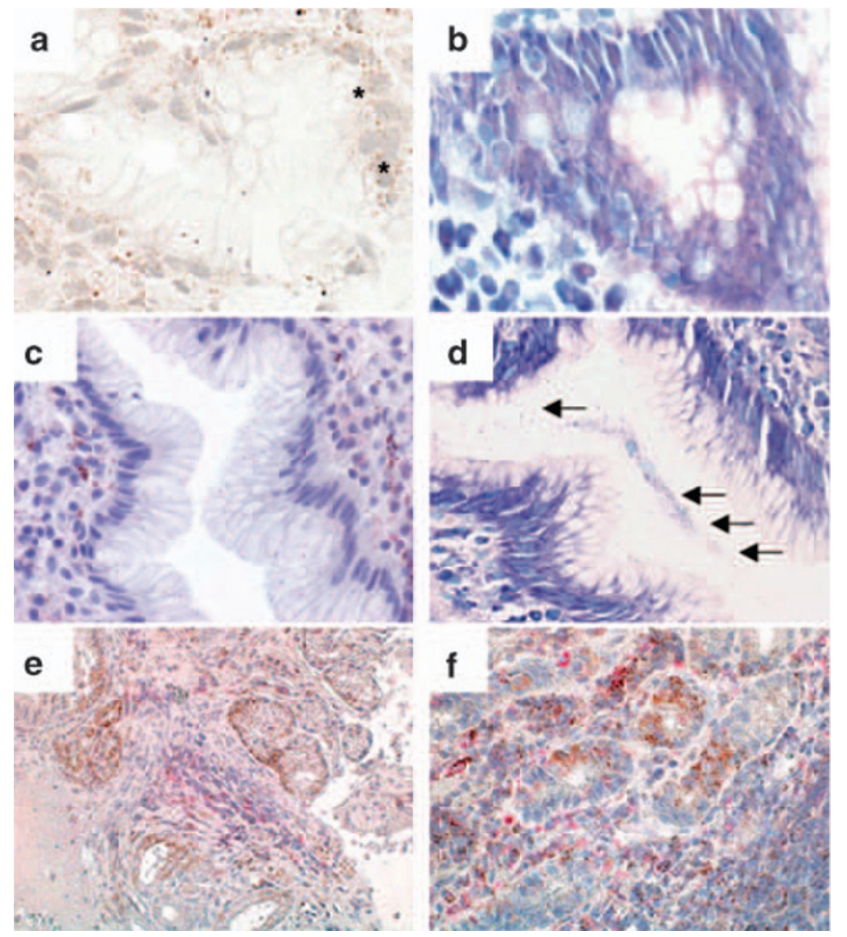

Figure 6 Localization of A PRoliferation-Inducing Ligand (APRIL) epithelial expression in $H$. Pylori gastritis. Sequential sections of $H$. Pylori gastritis stained for APRIL (a, c, e, and f) in single (brown $\mathbf{a}$ and $\mathbf{c}$ ) and double staining with CD20 (pink in e and f), as well as Giemsa staining to detect $H$. Pylori localization (b and d). Staining for APRIL and Giemsa on sequential sections of $H$. Pylori gastritis showing APRIL expression (a asterisks) in epithelium devoid of bacterial colonization (b), and no expression of the molecule in the epithelium (c) in areas heavily infected by the bacteria (d, arrows). Of interest, the epithelial areas positive for APRIL were consistently detected in close proximity to the organized, reactive lymphoid tissue (e and $\mathbf{f}$, arrows $\mathrm{CD} 20+$ cells in pink).

gene transcription independently of CD40/CD40L and TGF- $\beta{ }^{4}$ In this study, we showed that APRIL and AID in the gut are coexpressed in GALT, which is classically associated with T-cell-dependent CSR to IgA in GC. We showed that APRIL is expressed by macrophages and DCs in GC, in association with an unambiguous expression of AID. Therefore, GALT contains the molecular machinery to support both T-cell-independent and T-cell-dependent routes to CSR.

We showed that organized lymphoid tissue was the only microanatomical site in which APRIL and AID were coexpressed. AID induction and CSR require B-cell proliferation $^{19}$ and GALT contains two B-cell subsets, including dividing cells that could support these processes, a small percentage of marginal zone $\mathrm{B}$ cells and the highly proliferative GC B cells. Conversely, several reports have noted the absence of B-cell proliferation in lamina propria ${ }^{20}$ (data not shown). To our knowledge, there is no evidence that APRIL drives B-cell proliferation, thus suggesting that additional signals are needed in the microenvironment to support APRIL-induced CSR. The only place described so far, in which APRIL, AID, and cell division occur, is the GALT, an environment that we suggest could support both T-dependent and T-independent CSR. 
Lamina propria AID expression has been described in humans, ${ }^{7}$ alongside IgD + cells that are not a common feature of lamina propria. ${ }^{7}$ In contrast, all studies that did not detect lamina propria AID, ${ }^{20}$ such as this study, included a very careful definition of lamina propria with histology and IHC, and excluded samples containing CD20 + cells. This suggests that the discrepancies may be purely owing to definitions of microanatomical compartments, and false-positive results may be a consequence of sampling the periphery of GALT. Moreover, our analysis showed that in the lamina propria, the antibody commonly used for AID detection consistently binds to ceroidloaded macrophages and no AID/CD20 double-positive cells were detected in the lamina propria of all the tissues examined. Ceroid contains negatively charged phospholipids that may bind antibodies nonspecifically. It is important to acknowledge the nature of this artefact, which is not shared by a control rat antibody.

We detected APRIL in macrophages, DCs, and neutrophils in the lamina propria throughout the whole gastrointestinal tract. APRIL expression was associated with TACI and BCMA. Previous reports suggested that, in inflammatory conditions, crypt-infiltrating neutrophils provide APRIL to lamina propria plasma cells. ${ }^{15}$ In this paper, we show that crypt epithelial cells also express APRIL under physiological conditions. Thus, APRIL derived from epithelial and lamina propria cells potentially create a bone marrow-like survival niche for resident plasma cells in the gut. A similar pattern of APRIL expression has already been described in the murine lymph node, in which APRIL, together with IL- 6 and CXCL12, has been described in the medulla, in which it sustains plasmablast development. ${ }^{21}$ Increase of APRIL in the periphery of follicles might be associated with additional aspects of local differentiation of B cells. ${ }^{22}$

In the past few years, several reports linked the activation of receptors for pathogen-associated molecular patterns on epithelium with the release of B-cell survival factors and local B-cell activation..$^{23}$ In vitro, bacteria-stimulated colonic epithelial cells have been shown to produce APRIL on activation through TLR5. ${ }^{7}$ Given the ability of APRIL to induce IgA CSR, this mechanism has been advocated to justify the increase in IgA2 subclass production in colon, ${ }^{24}$ in which its short hinge region makes it more resistant to bacteria-derived protease. ${ }^{25}$ In this study, we show that APRIL is consistently expressed throughout the gastrointestinal tract, not only in the colon. Whereas bacteria antigen stimulation might preferentially induce IgA2 CSR, ${ }^{26}$ we propose that this occurs within the organized lymphoid tissue.

The observation of APRIL expression in crypts is consistent with a role for APRIL in epithelial cell turnover. Consistent with this hypothesis, malignant epithelium in gastric carcinoma, characterized by a high rate of cell proliferation (not only in crypts), generally shows intense APRIL staining by the malignant epithelium. In the epithelium, in close contact with GALT, whether constitutive or acquired in $H$. pylori gastritis, we detected a diffused APRIL expression toward luminal epithelium. This phenomenon suggests that APRIL induction may be modulated by immune cell-derived cytokines, highlighting a potential interaction between the diverse components of the gut immunological barrier.

To investigate the contribution of bacterial stimulation to physiological APRIL induction, we analyzed the epithelial expression of APRIL in the context of $H$. Pylori infection, as gastric epithelium is known to express TLR5 and $H$. pylori are flagellated bacteria. We could not detect any association between the presence of $H$. Pylori, mainly localized in the necks of gastric glands, and the epithelial expression of APRIL in any gastritis samples studied. This suggests that, if APRIL is induced on colonic epithelia by bacterial flora, other factors are also relevant, perhaps including antigen density or chronicity of challenge.

Overall, we showed that the T-independent IgA class switch machinery, comprising APRIL, its receptors, and AID, is expressed in GALT in physiological conditions, suggesting that GALT has the potential to support IgA induction through both T-dependent and T-independent mechanisms. We showed that APRIL is produced abundantly in lamina propria and in the epithelium providing a mucosal plasma cell survival niche. Our observation that the epithelial proliferation zone consistently expressed APRIL throughout the gut suggests that the property of APRIL, which provides the acronym 'A Proliferation-Inducing Ligand', may also be relevant to the maintenance of the mucosal barrier.

\section{METHODS}

Samples were taken from a collection of anonymous human tissues. Samples were obtained during therapeutic surgical resection or as diagnostic biopsies. Specimens were collected under the guidelines of the local research ethics committee and stored under license. Sequential sections of paraffin-embedded normal samples of stomach $(n=2)$, jejunum $(n=6)$, ileum $(n=8)$, colon $(n=9)$, appendix $(n=5)$, rectum $(n=2)$, and gastric carcinoma $(n=1)$, as well as $H$. pylori gastritis $(n=3)$, were used for immunohistochemical analysis. Frozen sections of ileum $(n=7)$, colon $(n=10)$, and palatine tonsil removed for sleep apnea $(n=6)$ were used for gene expression analysis.

\section{Immunohistochemistry}

All antibodies and reagents were purchased from Dako (Cambridge, UK), unless otherwise stated. Paraffin-embedded tissue sections $(3 \mu \mathrm{m})$ were submitted to antigen retrieval by heating in Dako Target Retrieval solution at $95^{\circ} \mathrm{C}$ for $40 \mathrm{~min}$. Endogenous peroxidase and alkaline phosphatase were blocked by means of a dual endogenous blocking solution. Sections were single and double stained for CD20 (1:20 dilution of the commercially provided stock), CD68 (1/50), Ki67 (1/50), CD11c (1/50), CD11b $(1 / 50)$, myeloperoxidase (1/100), elastase (1/50), and APRIL $(1 / 50)$ or AID (1/200) (Cell Signaling, Hitchin, Herts, UK) using the EnVision single and doublestaining systems according to the manufacturer's instructions. Rabbit anti-rat Igs were used as a secondary antibody for AID staining. After staining, sections were counterstained with hematoxylin. Isotype-matched control antibodies at appropriate concentrations were used as negative control. Sections of human tonsil were stained to provide a positive control.

Sequential sections of four samples of $H$. pylori-induced gastritis were stained with standard Giemsa staining to identify $H$. Pylori localization and were single/double stained with APRIL and CD20.

\section{Microdissection, mRNA isolation, qRT-PCR}

Microdissection and laser catapulting were performed on Cresyl-violet ( $0.1 \%$ in ethanol)-stained frozen tissue sections from ileum (7) and colon (10) samples, and on tonsil germinal centers as previously described. ${ }^{23}$ Messenger RNA (mRNA) was isolated from microdissected tissue 
(Qiagen RNAeasy, Crawley, West Sussex, UK) and reverse transcribed with a high-capacity reverse transcription kit (Applied Biosystems, Warrington, Cheshire, UK). Quantitative RT-PCR (Applied Biosystems) was performed as previously described ${ }^{17}$ for APRIL, BCMA, TACI, and AID mRNA expression. The codes for primers and probes used were as in the Applied Biosystems web site, APRIL (Hs00601664_g1), BCMA (Hs03045080_m1), TACI (Hs00234859_m1), and AID (Hs00757808_ m1, Hs00930003_g1, Hs00930002_g1, Hs00221068_m1), and were quantified with GAPDH (glyceraldehyde 3-phosphate dehydrogenase) (Hs99999905_m1) as an endogenous control. The quantitative real-time PCR was run in triplicate on a 384-well PCR plate (Applied Biosystems) and detected using an ABI PRISM 7900HT instrument. Results were analyzed with the ABI PRISM 7900HT Sequence Detection System Version 2.1 (SDS 2.3). $\Delta \mathrm{C}_{t}$ values were used for statistical analysis. The $\mathrm{C}_{t}$ value is the cycle number at which the exponential increase in fluorescence during the RT-PCR reaction crosses the threshold. We used the mean of three technical replicates $\left(C_{t}\right.$ values) to calculate the $\Delta C_{t}$ value that represents the $\mathrm{C}_{t}$ value for any sample normalized to the endogenous housekeeping gene. For each gene, preliminary experiments to set a common threshold were performed. $\mathrm{C}_{t}$ values above 34 were not accepted, neither were technical replicates with more than two cycle differences between them.

\section{Statistical analysis}

All statistical analyses were performed with GraphPad Prism version 3.03 for Windows (GraphPad Software, San Diego, CA). Data were analyzed with unpaired, two-sided $t$-tests. A $P$ value of $<0.05$ was considered statistically significant.

\section{ACKNOWLEDGMENTS}

This work was supported by a project grant from the BBSRC.

\section{DISCLOSURE}

The authors declare no conflict of interest.

() 2009 Society for Mucosal Immunology

\section{REFERENCES}

1. Hahne, M. et al. APRIL, a new ligand of the tumor necrosis factor family, stimulates tumor cell growth. J. Exp. Med. 188, 1185-1190 (1998).

2. Castigli, E. et al. Impaired IgA class switching in APRIL-deficient mice. Proc. Natl. Acad. Sci. USA 101, 3903-3908 (2004).

3. Bossen, C. \& Schneider, P. BAFF, APRIL and their receptors: structure, function and signaling. Semin. Immunol. 18, 263-275 (2006).

4. Litinskiy, M.B. et al. DCs induce CD40-independent immunoglobulin class switching through BLyS and APRIL. Nat. Immunol. 3, 822-829 (2002).

5. Shikina, T. et al. IgA class switch occurs in the organized nasopharynxand gut-associated lymphoid tissue, but not in the diffuse lamina propria of airways and gut. J. Immunol. 172, 6259-6264 (2004).

6. Bergqvist, P., Gardby, E., Stensson, A., Bemark, M. \& Lycke, N.Y. Gut IgA class switch recombination in the absence of CD40 does not occur in the lamina propria and is independent of germinal centers. J. Immunol. 177, 7772-7783 (2006).

7. He, B. et al. Intestinal bacteria trigger T cell-independent immunoglobulin A(2) class switching by inducing epithelial-cell secretion of the cytokine APRIL. Immunity 26, 812-826 (2007).
8. Fagarasan, S., Kinoshita, K., Muramatsu, M., Ikuta, K. \& Honjo, T. In situ class switching and differentiation to IgA-producing cells in the gut lamina propria. Nature 413, 639-643 (2001).

9. Brandtzaeg, P., Baekkevold, E.S. \& Morton, H.C. From B to A the mucosal way. Nat. Immunol. 2, 1093-1094 (2001).

10. Johansen, F.E. \& Brandtzaeg, P. Transcriptional regulation of the mucosal IgA system. Trends Immunol. 25, 150-157 (2004).

11. Tsuij, M. et al. Requirement for lymphoid tissue-inducer cells in isolated follicle formation and T cell-independent immunoglobulin A generation in the gut. Immunity 29, 261-271 (2008).

12. Kroese, F.G., de Waard, R. \& Bos, N.A. B-1 cells and their reactivity with the murine intestinal microflora. Sem. Immunol. 8, 11-18 (1996).

13. Carsetti, R., Rosado, M.M. \& Wardmann, H. Peripheral development of $B$ cells in mouse and man. Immunol. Rev. 197, 179-191 (2004).

14. Darce, J.R., Arendt, B.K., Wu, X. \& Jelinek, D.F. Regulated expression of BAFF-binding receptors during human B cell differentiation. J. Immunol. 179, 7276-7286 (2007).

15. Benson, M.J. et al. Cutting edge: the dependence of plasma cells and independence of memory B cells on BAFF and APRIL. J. Immunol. 180, 3655-3659 (2008).

16. Huard, B. et al. APRIL secreted by neutrophils binds to heparan sulfate proteoglycans to create plasma cell niches in human mucosa. J. Clin. Invest. 118, 2887-2895 (2008).

17. Bombardieri, M. et al. Activation-induced cytidine deaminase expression in follicular dendritic cell networks and interfollicular large B cells supports functionality of ectopic lymphoid neogenesis in autoimmune sialoadenitis and MALT lymphoma in Sjogren's syndrome. J. Immunol. 179, 49294938 (2007).

18. Schmausser, B. et al. Expression and subcellular distribution of toll-like receptors TLR4, TLR5 and TLR9 on the gastric epithelium in Helicobacter pylori infection. Clin. Exp. Immunol. 136, 521-526 (2004).

19. Rush, J.S., Liu, M., Odegard, V.H., Unniraman, S. \& Schatz, D.G. Expression of activation-induced cytidine deaminase is regulated by cell division, providing a mechanistic basis for division-linked class switch recombination. Proc. Natl. Acad. Sci. USA 102, 13242-13247 (2005).

20. Boursier, L., Gordon, J.N., Thiagamoorthy, S., Edgeworth, J.D. \& Spencer, J. Human intestinal IgA response is generated in the organized gut-associated lymphoid tissue but not in the lamina propria. Gastroenterology 128, 1879-1889 (2005).

21. Mohr, E. et al. Dendritic cells and monocyte/macrophages that create the IL-6/APRIL-rich lymph node microenvironments where plasmablasts mature. J. Immunol. 182, 2113-2123 (2009).

22. Bjerke, K. \& Brandtzaeg, P. Immunoglobulin- and J chain-producing cells associated with lymphoid follicles in the human appendix, colon and ileum, including Peyer's patches. Clin. Exp. Immunol. 64, 432-441 (1986).

23. Xu, W. et al. Epithelial cells trigger frontline immunoglobulin class switching through a pathway regulated by the inhibitor SLPI. Nat. Immunol. 8, 294-303 (2007).

24. Kett, K., Brandtzaeg, P., Radl, J. \& Haaijman, J.J. Different subclass distribution of IgA-producing cells in human lymphoid organs and various secretory tissues. J. Immunol. 136, 3631-3635 (1986).

25. Plaut, A.G., Wistar, R. Jr. \& Capra, J.D. Differential susceptibility of human IgA immunoglobulins to streptococcal IgA protease. J. Clin. Invest. 54 1295-1300 (1974).

26. Kett, K. et al. Intestinal B-cell isotype response in relation to local bacterial load: evidence for immunoglobulin A subclass adaptation. Gastroenterology 109, 819-825 (1995). 